\title{
NOISY PHASE UNWRAP FOR FRINGE TECHNIQUES: ADAPTIVE LOCAL POLYNOMIAL APPROXIMATIONS
}

\author{
Vladimir Katkovnik, Jaakko Astola, Karen Egiazarian \\ Signal Processing Institute, University of Technology of Tampere, \\ P. O. Box 553, Tampere, Finland. E-mail: katkov@cs.tut.fi.
}

\begin{abstract}
Many imaging systems deal with phase measurements using coherent radiation in order to illuminate objects. The reflected scattered return carries information on physical and geometrical properties of illuminated objects. It can be information on shape, deformation, movement and structure of the object's surface. We propose a novel phase unwrap filtering for noisy holographic data. Based on the window size adaptive local polynomial approximation it allows dramatically reduce level of noise and in the same time preserve phase variation features. Simulation shows that the technique enables an advanced accuracy for phase reconstruction from wrapped noisy observations.
\end{abstract}

Index Terms - Fringe techniques, hologram, local polynomial approximation, phase unwrap, speckle interferometry.

\section{INTRODUCTION}

A variety of imaging systems deal with phase measurements using coherent radiation in order to illuminate objects. The reflected scattered return carries information on physical and geometrical properties of the illuminated objects. It can be information on shape, deformation, movement and structure of the object's surface.

Common to these applications is that the observations are periodical functions of the phase which can be interpreted as the principal phase value defined on the interval $[-\pi, \pi)$. Accordingly, it is impossible to unambiguously reconstruct the original, nonrestricted values, hereafter referred to as the true phase, unless additional assumptions are introduced. If a true phase value is outside the principal interval $[-\pi, \pi)$, the observed value is wrapped into this interval, corresponding to an addition or subtraction of an integer number of $2 \pi$. Many approaches start from estimation of the phase for the principal interval and further extend these estimates to nonrestricted values. This last procedure is known as phase unwrapping. What make this problem even more difficult is that the measured values are usually corrupted by noise.

The standard formulation of the phase unwrapping starts from the observation model in the form $z_{\phi}=W\left(\varphi+n_{\varphi}\right)$, where $\varphi$ is the true phase, $n_{\varphi}$ is a random noise additive to the phase $\varphi, z_{\phi}$ is the observed phase. Here $W$ is a wrapping operator transforming the noisy phase to the basic phase interval $[-\pi, \pi)$. There is an obvious link between the wrapped $\phi$ and non-wrapped true phase $\varphi$

$$
\varphi=\phi+2 \pi k, \phi \in[-\pi, \pi),
$$

where $k$ is an integer. The wrapping operator is equivalent to division by module $2 \pi, \phi=\bmod \{\varphi+\pi, 2 \pi\}-\pi$, which separate $\varphi$ on two parts: fractional and integer parts. The latter is defined as $2 \pi k$ with an integer $k$. The basic unwrapping problem is to reconstruct $\varphi(x, y), x, y \in X$, from the observations $z_{\phi}(x, y), x, y \in X$. In our simulation we assume that $X$ is an integer $2 D$ grid, $X=\left\{x, y: x=1,2, \ldots N_{1}\right.$, $\left.y=1,2, \ldots N_{2}\right\}$. The equation (1) shows that there is no oneto-one relation between the wrapped and unwrapped phase. Surprisingly, differentiation of the observations $z_{\varphi}$ can resolve this ambiguity or at least to reduce it dramatically.

The techniques developed for phase unwrapping can be roughly separated in two large classes. The algorithms of the first class use a two stage approach with estimation of the gradient at the first stage and the following integration of this gradient at the second stage. There are two main difficulties in this approach. First, the non-alising sampling conditions sufficient for perfect reconstruction of the phase from the gradient:

$$
\begin{gathered}
-\pi \leq \Delta_{x} \varphi(x, y)<\pi,-\pi \leq \Delta_{y} \varphi(x, y)<\pi \\
\Delta_{x} \varphi(x, y)=\varphi(x, y)-\varphi(x-1, y) \\
\Delta_{y} \varphi(x, y)=\varphi(x, y)-\varphi(x, y-1)
\end{gathered}
$$

often are not fulfilled for noisy phase $\phi$. Then smoothness assumptions imposed on the true phase are used for regularization of the problem in order to avoid the aliasing affects. Second, numerical differentiation as well as numerical integration are not trivial operation for noisy data as the noise suppression should be incorporated. In particular, the differentiation results in increasing the noise level and the integration is an inverse ill-conditined operation.

The algorithms of the second class are based on direct approximation of the reconstructed phase. In this way the differentiation as well as the integration are completely avoided. 
This sort of algorithms are potentially can give more accurate results and demonstrate a better noise resistance. However, the price is more expensive calculations based on recursive solution of nonlinear estimation equations. A comprehensive review of the phase unwrap problems and algorithms is given in [1]. This paper is devoted to the approach belonging to the second class of the algorithms.

\section{PROPOSED PHASE-APPROXIMATION METHOD}

The approach develops in this paper is based on two independent ideas: local approximation for design of nonlinear filters (estimators) and adaptation of these filters to unknown smoothness of the varying phase. As flexible universal tools we use local polynomial approximation ( $L P A)$ for approximation and intersection of confidence intervals $(I C I)$ for adaptation [2].

The $L P A$ is applied for filter design using a polynomial fit in a sliding window. The window size as well as the order of the polynomial define a desirable filter. The window size is considered as a varying adaptation parameter of the filter.

The $I C I$ is an adaptation algorithm. It searches for a largest local window size where the variance and the bias of the estimates are balanced. It is shown that the $I C I$ adaptive $L P A$ is efficient and allows to get a nearly optimal quality of estimation. To deal with anisotropic phases including sharp elements nonsymmetric directional windows can be used. The corresponding directional (multidirectional) LPA filters equipped with the $I C I$ adaptive window sizes demonstrate an advanced performance. The $L P A-I C I$ algorithms are recent powerful tools in signal and image processing. These algorithms demonstrate the state-of-art performance and on many occasions visually and quantitatively outperform some of the best existing methods. The $L P A-I C I$ techniques define a wide class of pointwise spatially/time adaptive filters applicable in many scientific and engineering fields [2]. There is a variety of phase observation models depending on measurement principals.

Here we wish to mention two basic ones:

(1) Sine/cosine observations

$$
z_{1}=\cos \varphi+n_{z_{1}}, z_{2}=\sin \varphi+n_{z_{2}}
$$

with the phase calculated as $\phi=\arctan \frac{z_{1}}{z_{2}}$.

(2) Phase-shifting observations $z_{l}=A_{0}+A_{1} \cos (\varphi+$ $\left.\delta_{l}\right)+n_{z_{l}}, l=1, \ldots, L$, where $\delta_{l}$ are fixed shifted phases, $A_{0}$ is a background intensity, and $A_{1}$ is an amplitude of the harmonic phase function.

One of the most popular choices is $\delta_{l}=(l-1) \pi / 2, l=$ $1,2,3,4$. Then, the intensities $A_{0}, A_{1}$ and the phase $\varphi$ can be found with the formula for the phase $\phi=\arctan \frac{z_{4}-z_{2}}{z_{1}-z_{3}}$. It is obvious that the above formulas for $\varphi$ are accurate provided that the observations are noiseless. Note also that these formulas give the wrapped phase belonging to the segment $[-\pi, \pi)$. Thus, we have for these models the observations $z_{\phi}$ respectively as $z_{\phi}=\arctan \frac{z_{1}}{z_{2}}$ and $z_{\phi}=\arctan \frac{z_{4}-z_{2}}{z_{1}-z_{3}}$.

A number of phase-shifting observations with different phase shifts and different number of observations can be see in [3]. This sort of models for the phase can be represented in the form as $z_{\phi}=W\left(\varphi+n_{\varphi}\right)$ but the additive noise $n_{\varphi}$ in these cases is signal dependent. Then this model with the signal independent additive noise $n_{\varphi}$ is quite approximate, however, giving convenient tool for simulation and theoretical study of the signal processing methods.

In our approach we assume that the observed data are already in the form (3). Then we calculate $\cos \left(z_{\phi}\right)=\cos (\varphi+$ $\left.n_{\varphi}\right)$ and $\sin \left(z_{\phi}\right)=\sin \left(\varphi+n_{\varphi}\right)$, and apply $L P A$ in order to approximate $\varphi$ as argument of these harmonic functions. In principal the approach can be applied in order to approximate $\varphi$ in the argument of the wrap operator. However, this operator is discontinuous with respect to $\varphi$ and use the harmonic transform allows to replace it by the smooth differentiable sine and cosine functions.

\section{SLIDING LPA}

Let us recall basic ideas of $L P A$ (e.g. [2]) and introduce the $L P A$ estimates of the phase. Assume that in some neighborhood of the point $(x, y)$ the phase $\varphi(x, y)$ can be represented in the form

$$
\tilde{\varphi}\left(x_{s}, y_{s} \mid \mathbf{c}\right)=p^{T}\left(x_{s}, y_{s}\right) \mathbf{c}
$$

where $p=\left(p_{1}, p_{2}, p_{3}\right)$ is a vector of the first order polynomials $p_{1}=1, p_{2}=x, p_{2}=y$, and $\mathbf{c}=\left(c_{1}, c_{2}, c_{3}\right)^{T}$ is a vector of unknown parameters. The loss function of the local fit is

$$
\begin{array}{r}
L_{h}(x, y, \mathbf{c})= \\
\frac{1}{2} \sum_{s} w_{h, s}\left\{\left[z_{1}\left(x+x_{s}, y+y_{s}\right)-\cos \tilde{\varphi}\left(x_{s}, y_{s} \mid \mathbf{c}\right)\right]^{2}+\right. \\
\left.\left[z_{2}\left(x+x_{s}, y+y_{s}\right)-\sin \tilde{\varphi}\left(x_{s}, y_{s} \mid \mathbf{c}\right)\right]^{2}\right\}, \\
w_{h, s}=w_{h}\left(x_{s}, y_{s}\right) \geq 0,
\end{array}
$$

and the parameter $\mathbf{c}$ is defined as follows

$$
\hat{\mathbf{c}}(x, y)=\arg \min _{\mathbf{c}} L_{h}(x, y, \mathbf{c}) .
$$

The $L P A$ estimates of the signal and the first derivatives are as follows [2]

$\hat{\varphi}(x, y)=\hat{\mathbf{c}}_{1}(x, y), \hat{\varphi}_{x}^{(1)}(x)=\hat{\mathbf{c}}_{2}(x, y), \hat{\varphi}_{y}^{(1)}(x)=\hat{\mathbf{c}}_{3}(x, y)$

Thus the estimates are defined by the nonlinear least square method with the loss function quadratic with respect to the errors of approximation. The window $w_{h, s}$ defines a set of neighborhood observations and their weights in estimation for $(x, y)$. The window size (scale) parameter $h$ in $w_{h}$ defines the size of the window and usually used in the form 
$w_{h}(x, y)=w(x / h, y / h), h>0$. In particular for the square uniform window $w=1$ for $|x| \leq 1,|y| \leq 1$ and $w=0$ otherwise. It means that $w_{h}=1$ for $|x| \leq h,|y| \leq h$ and $w_{h}=0$ otherwise. A smaller or larger $h$ narrows or widens the window $w_{h}$, respectively.

The window function $w_{h}$ can be symmetric or non-symmetric with respect the origin point $x=0, y=0$. The formula (5) shows that we obtain simultaneously the estimates of the phase $\hat{\varphi}$ and the instantaneous spatial frequencies $\hat{\varphi}_{x}^{(1)}$ and $\hat{\varphi}_{y}^{(1)}$. In general, the estimate $\hat{\mathbf{c}}(x, y)$ depends of the coordinates $(x, y)$ and the window size $h$. We wish to emphasize the nonparametric nature of the introduced estimates. Indeed we start from the parametric linear on $x$ and $y$ model in (4) and could expect that the approximation (estimate) is also linear on $x$ and $y$. However, the fit is used in the polynomial approximation (4) only for one "central" point $x_{s}=y_{s}=0$ [2]. For the phase it gives $\hat{\varphi}(x, y)=\tilde{\varphi}(0,0 \mid \mathbf{c})=\hat{\mathbf{c}}_{1}(x, y)$, and for the derivatives $\hat{\varphi}_{x}^{(1)}(x, y)=\partial \tilde{\varphi}(x, y \mid \mathbf{c}) /\left.\partial x\right|_{x=0, y=0}=\hat{\mathbf{c}}_{2}(x, y)$, $\hat{\varphi}_{y}^{(1)}(x, y)=\partial \tilde{\varphi}(x, y \mid \mathbf{c}) /\left.\partial y\right|_{x=0, y=0}=\hat{\mathbf{c}}_{3}(x, y)$. The result of this point-wise use of the approximation is that the parametric estimate (4) becomes nonparametric ones with. $\hat{\varphi}(x, y)$ nonlinear with respect to $x$ and $y$.

All ideas of the standard $L P A$ concerning the window $w$ (shape, anisotropy, directionality, etc.), the scaling $h$ (scalar, multivariate), estimation of the signal and derivatives are naturally valid in this nonparametric pointwise estimation.

\subsection{Point-wise estimate calculation}

Minimization of $L_{h}(x, y, \mathbf{c})$ non-quadratic with respect to $\mathbf{c}$ cannot be expressed in an explicit analytical form and requires numerical recursive calculations using the vector-gradient $\partial_{\mathbf{c}} L_{h}(x, y, \mathbf{c})=\left(\partial_{c_{i}} L_{h}(x, y, \mathbf{c})\right)_{M \times 1}$ and the second derivatives (Hessian matrix) of $L_{h}, \partial_{\mathbf{c}} \partial_{\mathbf{c}^{T}} L_{h}(x, y, \mathbf{c})=$ $\left(\partial_{c_{i}} \partial_{c_{j}} L_{h}(x, y, \mathbf{c})\right)_{M \times M}$. Here we use $M$ to denote the dimension of the vector $\mathbf{c}$. In the considered particular case $M=3$, however using higher order $L P A$ may lead to larger $M$.

A gradient descent recursive procedure has a standard form (e.g. [4])

$$
\mathbf{c}^{(k+1)}=\mathbf{c}^{(k)}-\gamma_{k} A^{(k)} \partial_{\mathbf{c}} L_{h}\left(x, y, \mathbf{c}^{(k)}\right), k=0,1, \ldots,
$$

where $\mathbf{c}^{(k)}$ are successive iterations of $\mathbf{c}$, and the gradient $\partial_{\mathbf{c}} L_{h}$ is calculated for $\mathbf{c}=\mathbf{c}^{(k)}$.

The possible procedures are different by an $M \times M$ weight matrix $A^{(k)}$ and a step size parameter $0<\gamma_{k} \leq 1$. We found that the Gauss-Newton version of the algorithm works very well. The $A^{(k)}$ in (6) is an approximation of the inverse Hessian matrix computed using the first derivatives of $y$ only. For the Gauss-Newton method the matrix $A$ in (6) is calculated as follows. First we produce linearization of $\sin \tilde{\varphi}\left(x_{s}, y_{s} \mid \mathbf{c}+\delta \mathbf{c}\right)$ and $\cos \tilde{\varphi}\left(x_{s}, y_{s} \mid \mathbf{c}+\delta \mathbf{c}\right)$ assuming that $\tilde{\varphi}\left(x_{s}\right.$, $p^{T}\left(x_{s}, y_{s}\right) \mathbf{c}+p^{T}\left(x_{s}, y_{s}\right) \delta \mathbf{c}$, where $\delta \mathbf{c}$ is a small variation of c:

$$
\begin{array}{r}
\sin \tilde{\varphi}\left(x_{s}, y_{s} \mid \mathbf{c}+\delta \mathbf{c}\right)= \\
\sin \tilde{\varphi}\left(x_{s}, y_{s} \mid \mathbf{c}\right)+\cos \tilde{\varphi}\left(x_{s}, y_{s} \mid \mathbf{c}\right) \cdot p^{T}\left(x_{s}, y_{s}\right) \delta \mathbf{c}, \\
\cos \tilde{\varphi}\left(x_{s}, y_{s} \mid \mathbf{c}+\delta \mathbf{c}\right)= \\
\cos \tilde{\varphi}\left(x_{s}, y_{s} \mid \mathbf{c}\right)-\sin \tilde{\varphi}\left(x_{s}, y_{s} \mid \mathbf{c}\right) \cdot p^{T}\left(x_{s}, y_{s}\right) \delta \mathbf{c} .
\end{array}
$$

Substitute these series in $L_{h}(x, y, \mathbf{c})$, then the matrix corresponding to Gauss-Newton method is defined as

$$
\hat{H}=\partial_{\delta \mathbf{c}} \partial_{\delta \mathbf{c}^{T}} L_{h}=\sum_{s} w_{h, s} p\left(x_{s}, y_{s}\right) p^{T}\left(x_{s}, y_{s}\right)
$$

and in (6) $A=\hat{H}^{-1}$.

\subsection{Algorithm organization}

Initialization is an important element of the above point-wise phase estimation. The unwrapped phase image reconstruction is organized as the recursive procedure over the phase image using for initialization the estimates already obtained for neighboring points. Let us introduce some sequence $\left\{x^{(n)}, y^{(n)}\right\}$ of the neighboring points of the phase image. It can be done in many different way. The simplest is a line-byline sequence starting from the pixel $(1,1)$ and going along the first line as $(1,2),(1,3), \ldots,\left(1, N_{2}\right)$, further the pixels of the second line $(2,1),(2,2), \ldots,\left(2, N_{2}\right)$, and in a similar way up to the last line $\left.\left(N_{1}, 1\right), N_{1}, 2\right), \ldots,\left(N_{1}, N_{2}\right)$. This procedure orders the pixel of the image as $\left\{x^{(n)}, y^{(n)}\right\}_{n=1, \ldots N_{1} N_{2}}$. Let $\mathbf{c}^{(n)}\left(x^{(n)}, y^{(n)} \mid \mathbf{c}\right)$ be the estimate for the point $\left(x^{(n)}, y^{(n)}\right)$ provided that the recursive estimate algorithm (6) is initiated by the vector $\mathbf{c}$. Then overall the proposed algorithm can be given in the following recursive form

$$
\begin{aligned}
& \mathbf{c}^{(n)}=\mathbf{c}^{(n)}\left(x^{(n)}, y^{(n)} \mid \mathbf{c}^{(n-1)}\right), \\
& n=2, \ldots, N_{1} \cdot N_{2}, \\
& \hat{\varphi}\left(x^{(n)}, y^{(n)}, h\right)=c_{1}^{(n)}, \\
& \hat{\varphi}_{x}^{(1)}\left(x^{(n)}, y^{(n)}, h\right)=c_{2}^{(n)}, \hat{\varphi}_{y}^{(1)}\left(x^{(n)}, y^{(n)}, h\right)=c_{3}^{(n)} .
\end{aligned}
$$

Note that this recursive procedure includes the recursive point-wise estimation (6) as imbedded one.

The procedure (8) is initiated by the estimate for the first point $\left(x^{(1)}, y^{(1)}\right)$. This estimate $c^{(1)}$ can be defined by boundary conditions for the reconstructed function, a prior information or by a rough estimate obtained by another method.

\subsection{Estimate accuracy and adaptation}

The estimation accuracy is characterized by errors defined as a difference between the true phase value and the correspondying-efitimate. These errors are composed of the systematic (bias) and random components corresponding to the deterministic $\varphi$ and the random noise $\varepsilon$, respectively. 
The scale $h$ is a crucial parameter for the accuracy of estimation. When the scale $h$ is relatively small, the $L P A$ gives a good smooth fit of signals but then fewer number of observations are used and the estimates are more variable and sensitive with respect to the noise. The best choice of $h$ involves a trade-off between the bias and variance, which depends on the degree of the $L P A$, a sample period, the noise variance and the derivatives of $y$ of the orders beyond the degree used in the LPA. The intersection of confidence interval $(I C I)$ rule is used for adaptive selection of $h$ close to the optimal one. The estimates are calculated for a set of different values of $h$ and the $I C I$ algorithm select the best one on the base of the hypothesis testing procedure [2].

\section{SIMULATION EXPERIMENTS}

Here we demonstrate the performance of the algorithm in application scenario typical for deformation (surface) measurements. The observations are given by $\sin / \cos$ model $z_{1}=$ $\cos \varphi+n_{z_{1}}, z_{2}=\sin \varphi+n_{z_{2}}$, where the phase $\varphi$ is a true unknown "pyramid" function (Fig.1, first image). The noise is white gaussian with independent $n_{z_{1}}$ and $n_{z_{2}}$ having standard deviation $\sigma$. In Fig.1 we can see also the noisy wrapped data calculated as $\phi=\arctan \frac{z_{1}}{z_{2}}$. The last image illustrates the highly noisy input data of the algorithm.

Figure 1 shows the result: the estimate of the phase $\varphi$. Comparing with the corresponding true unwrapped phase we can conclude that the obtained estimate is very accurate. The last image in Figure 1 shows the distribution of the estimation errors.

For estimation we use a set of square symmetric neighborhoods of the size $(2 h+1)$ with $h=0,1,2,3,4$. The $I C I$ algorithm gives the adaptive window sizes shown in Figure 2. This algorithm selects the large values of $h$ for the points which are far from the edges of the pyramid and the smallest for the edges and the points close to the edges. In this way the noise in the data is filtered very well and the edges of the pyramid are well preserved, while naturally the random errors at these edges are higher than it is for the plane areas of the pyramid (see the last image in Figure 1). Numerically, the estimation accuracy is characterized by $R M S E=0.075$ (root-mean-square error) and $M E D=0.03$ (median of errors).

A simulation study produced for many test functions shows state-of-the-art accuracy of the developed algorithm overcoming best algorithms known in the field. In particular, we produced a comparative study of our algorithm with respect to the $Z \pi M$ algorithm recently proposed in [5]. This last algorithm demonstrates very good results and can be treated as a bench mark in the field. We show that on many occasions our algorithm demonstrates a better performance.

\section{REFERENCES}

[1] D. C. Ghiglia and M. D. Pritt, Two-Dimensional Phase Unwrapping: Theory, Algorithms, and Software. New York: Wiley, 1998.

[2] V. Katkovnik, K. Egiazarian, J. Astola, Local Approximation Techniques in Signal and Image Processing. SPIE PRESS, Bellingham, Washington, 2006.

[3] Th. Kreis, Handbook of Holographic Interferometry (Optical and Digital Methods), Wiley-VCH Gmbh\&Co.KGaA, Weinheim, 2005.

[4] Fletcher R. Practical methods of optimization. John Wiley \& Sons, Inc. New York, 1987.

[5] J. Dias and J. Leitao, "The $Z \pi M$ algorithm: a method for interferometric image reconstruction in SAR/SAS," IEEE Trans Image Processing, vol. $11, \mathrm{n}^{0} 4$, pp. 408$422,2002$.
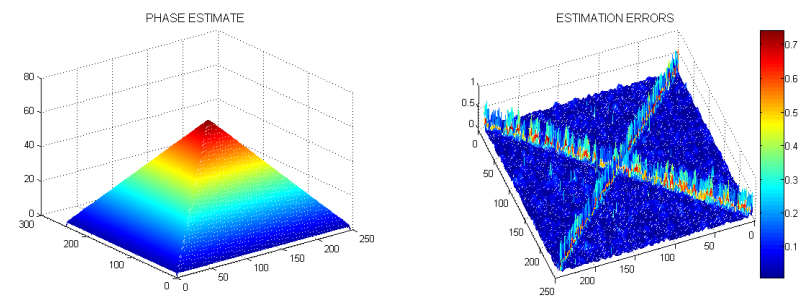

Fig. 1. True phase, noisy phase observations, estimate and absolute estimation errors given in radians.

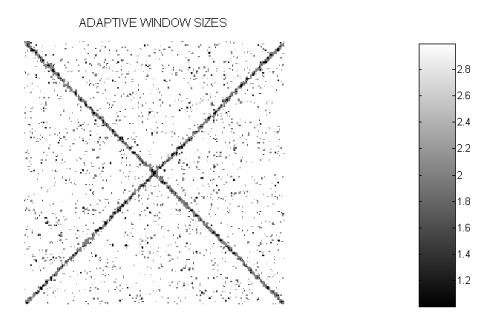

Fig. 2. Adaptive window sizes obtained by the ICI rule. 